\title{
On Near-Horizon Maximum Brightness of Cloudless Sky
}

\author{
V. V. Bakhanov, A. A. Demakova, V. I. Titov* \\ Federal Research Center Institute of Applied Physics, Russian Academy of Sciences, \\ Nizhny Novgorod, Russian Federation \\ *e-mail: titov@hydro.appl.sci-nnov.ru
}

\begin{abstract}
The brightness angular structure of the cloudless sky is studied based on the model of the sunlight single scattering. It is shown that the so-called near-horizon maximum of the sky brightness can be described within the framework of this model. Physical mechanism of arising of this maximum is analyzed; dependence of the maximum position on the light wavelength is explained. When the light wavelength increases, the sky brightness maximum "shifts" towards the horizon. It is related to the fact that the atmosphere optical thickness decreases with growth of a wavelength. These model data are compared to the experimental angular characteristics of the sky brightness obtained due to digital imaging of the horizon from the oceanographic platform. Possibility of estimating the atmosphere optical thickness using the angular position of the sky brightness near-horizon maximum is analyzed. Proposed is the algorithm for assessing the given characteristic for a certain value of the light wavelength based on graphical "inversion" of dependence of the angular distribution of the cloudless sky brightness upon the atmosphere optical thickness. The proposed algorithm and the horizon digital images permit to assess the atmosphere optical thickness in the R, G and B spectral ranges. The algorithm "robustness" to the errors in determining the sun azimuth relative to the observer is analyzed. The obtained estimates of the atmosphere optical thicknesses are in agreement with the known results of the nature measurements of the atmosphere analogous characteristics. The described method for reconstructing the values of the atmosphere optical thicknesses makes it possible to develop the applied sky brightness model taking into account multiple light scattering. The obtained values of optical thickness can be used in the models of angular distribution of the cloudless sky brightness to provide possibility of estimating the waves' statistical characteristics by the remote optical method.
\end{abstract}

Keywords: atmosphere, optics, optical thickness, sky brightness, sun, scattering of light, single scattering, aerosol, wavelength of light, cloudless sky, remote sensing.

Acknowledgements: the research is carried out at support of the RFFI grant No.16-05-00858a.

For citation: Bakhanov V. V., Demakova A. A., Titov V. I., 2018. On Near-Horizon Maximum Brightness of Cloudless Sky. Physical Oceanography, [e-journal] 25(6), pp. 437-447. doi:10.22449/1573-160X-2018-5-437-447

DOI: $10.22449 / 1573-160 X-2018-6-437-447$

(c) 2018, V. V. Bakhanov, A. A. Demakova, V. I. Titov

(C) 2018, Physical Oceanography

\section{Introduction}

As is known, information on the angular structure of the sky brightness is necessary for remote optical diagnostics of the sea surface, particularly, to restore the wave spectra from the sea surface images, to obtain data on the variability of the wave characteristics in oil slicks, internal waves and wind gusts on the sea surface [1-3]. There are model approximations of the angular distribution of the cloudless sky brightness, which require knowledge of the optical thickness of the atmosphere. As a rule, to describe the sunlight scattering in the atmosphere, the approximation of its single or double scattering by atmospheric aerosol is applied [4-8]. The difficulty in describing the brightness of the daytime sky consists in the great variation of the optical characteristics of the atmosphere. Due to this fact, the prob- 
lem to obtain data on the optical characteristics of the atmosphere in real time using simple tools arises.

Radiometric, polarimetric, lidar and satellite methods for determining the optical characteristics of the atmosphere and atmospheric aerosol have been recently developed [4, 9-18]. These methods require special equipment and do not allow receiving data in real time. In the present paper a method for determining the optical thickness of the atmosphere in three spectral ranges of light from the angular height of the horizontal maximum brightness of a cloudless sky recorded with a digital camera is proposed. This method provides real-time estimates of the atmospheric optical thicknesses for three spectral ranges of light $R, G$ and $B$. The obtained optical thickness values can be used in models of the angular distribution of the cloudless sky brightness to estimate the statistical characteristics of the optical waves as well as to study the optical properties of the atmosphere.

The present article is aimed to apply data on the near-horizontal maximum of sky brightness recorded by a digital camera to obtain information on the optical thickness of the atmosphere in three spectral ranges of light.

\section{Cloudless Sky Brightness Model}

The angular distribution of the spectral density of the sky brightness [16] (of $\mathrm{W} / \mathrm{m}^{2} \cdot \mathrm{nm}$ sterad dimension), which for brevity sake will be further noted as sky brightness, in the approximation of single scattering of non-polarized sun light in the plane-parallel non-absorbing atmosphere model will be defined by the following formulas [4-6]:

$$
\begin{gathered}
I=\pi S_{\lambda} \frac{f_{n}(\varphi)}{4 \pi} P\left(z, z_{s}\right), \\
P\left(z, z_{s}\right)=\frac{\exp (-\tau / \cos z)-\exp \left(-\tau / \cos z_{s}\right)}{\cos z / \cos z_{s}-1},
\end{gathered}
$$

where $\pi S_{\lambda}$ is the tabulated spectral solar constant, which dimension coincides with the dimension of the spectral density of the sky brightness; $z$ and $z_{s}$ are zenith distance of the sky and sun points; $\varphi$ is the scattering angle; $f_{n}(\varphi)$ is the dimensionless normalized light scattering indicatrix, which is the sum of Rayleigh and aerosol scattering indicatrices:

$$
f_{n}(\varphi)=\frac{\tau_{r}}{\tau} \frac{3}{4}\left(1+\cos ^{2} \varphi\right)+\frac{\tau_{a}}{\tau} 0.34 \frac{1+\cos ^{2} \varphi}{1-\cos \varphi},
$$

here $\tau=\tau_{r}+\tau_{a}$ is the dimensionless optical thickness of the atmosphere; $\tau_{r}$ and $\tau_{a}$ are the dimensionless Rayleigh and aerosol thickness of the atmosphere, whose values depend on the wavelength of light.

The normalized aerosol indicatrix of light scattering in the atmosphere is expressed by $0.34 \frac{1+\cos ^{2} \varphi}{1-\cos \varphi}$. It has various approximations [15, 16]. Factor 
$P\left(z, z_{s}\right)$ describes a decrease of the direct sunlight brightness to and along the observation line as it propagates to the observer according to the Bouguer law.

The scattering angle $\varphi$ is determined by the following formula

$$
\cos \varphi=\cos z_{s} \cos z+\sin z_{s} \sin z \cos \psi,
$$

where $\psi-$ is the angle between the projections on the horizontal plane of vectors directed to the sky and the sun or the azimuth angle of the sun relative to the observer.

The dependences of optical thickness on the light wavelength will be given in the following form [16]:

$$
\begin{aligned}
& \tau_{r}=\tau_{r}(550)(550 / \lambda)^{4}, \\
& \tau_{a}=\tau_{a}(550)(550 / \lambda)^{0,7}
\end{aligned}
$$

where $\lambda(\mathrm{nm})$ is the light wavelength. Values of optical thickness for light with a wavelength of $550 \mathrm{~nm}$ are set equal to $\tau_{r}(550)=0.098, \tau_{a}(550)=0.33$. The exponent in the formula for $\tau_{a}$ (the so-called Angstrom exponent) corresponds to the atmospheric transparency mean value for the coastal areas of the ocean. According to the data of [16], aerosol optical thickness $\tau_{a}(550)$ above the ocean can vary within the range of $0.04-0.4$, at that its mean value is 0.19 .

Fig. 1 shows that with increasing wavelength of light, optical thickness values decrease.

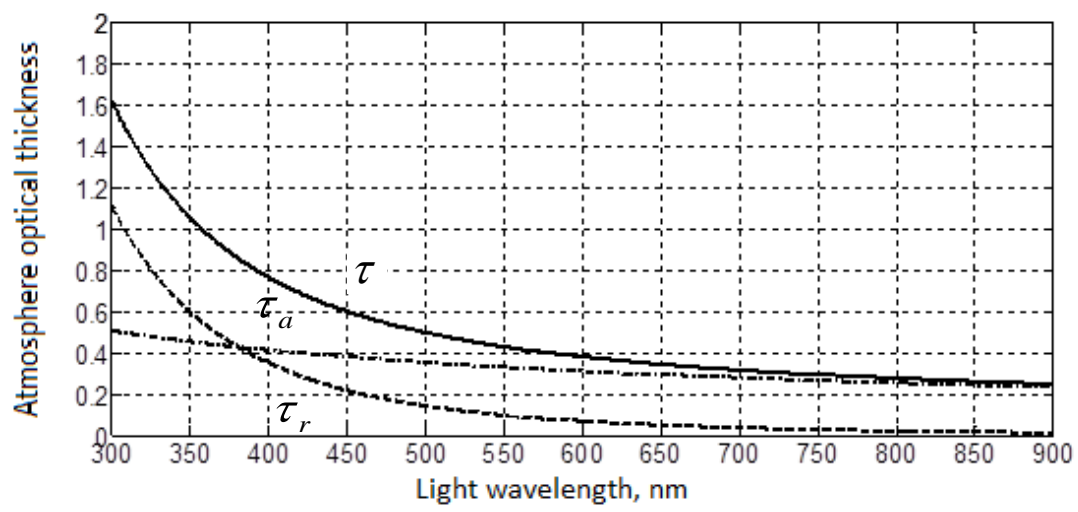

F i g. 1. Dependences of the atmosphere optical thicknesses upon the light wavelength. Indications: $\tau_{r}$ is the Rayleigh optical thickness; $\tau_{a}$ is the aerosol optical thickness; $\tau$ is the sum of the aerosol and the Rayleigh optical thickness

\section{Angular Distributions of a Cloudless Sky Brightness}

We shall consider the sky brightness distributions calculated from the model above. Fig. 2 shows the dimensionless normalized to the spectral solar constant sky brightness $I / \pi S_{\lambda}$ (hereinafter referred to as the normalized sky brightness) in the opposite to sun direction ( $\psi=180^{\circ}$ ). The sun zenith angle is hereinafter assumed to be equal to $60^{\circ}\left(z_{s}=60^{\circ}\right)$ in all the calculations. In the area of the sky zenith angle 
of $30^{\circ}$, there is a so-called solar point, where the sky brightness values are minimal. At this point, the angular distance to the sun is about $90^{\circ}$. This minimum is due to the minimum value of the scattering indicatrix at a scattering angle of $90^{\circ}$. At large zenith angles (near the horizon) there is a maximum sky brightness for all wavelengths of light.

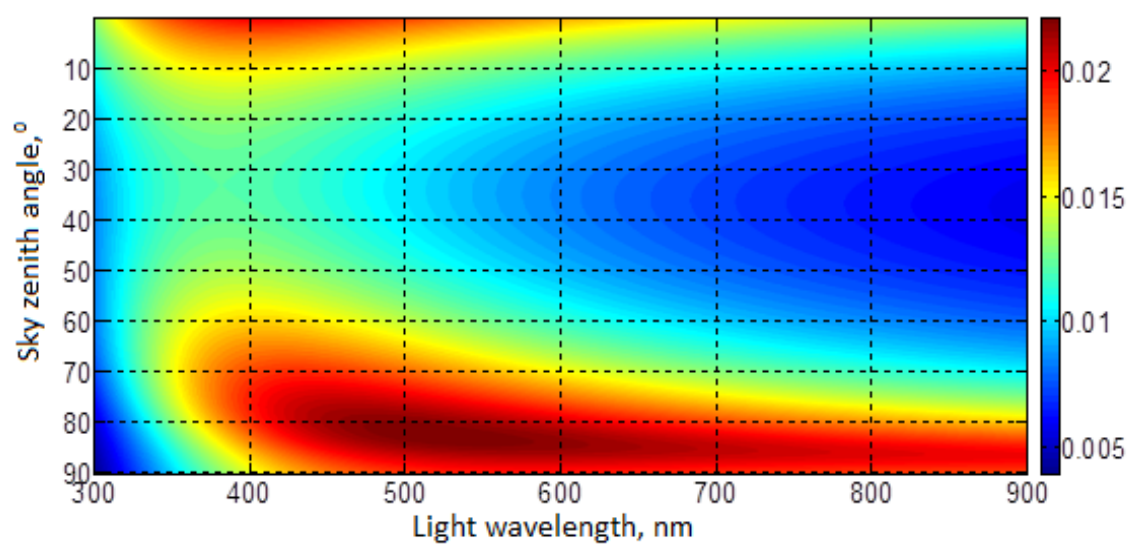

F i g. 2. The normalized sky brightness in the opposite to the sun direction as a function of the sky zenith angle $z$ and the light wavelength. Here and in the Fig. 5, 8 and 10, the normalized brightness value is shown in the conventional color scale

Fig. 3 shows the dimensionless normalized sky brightness for the wavelengths of light 450, 520 and $670 \mathrm{~nm}$, which correspond to the $R, G$ and $B$ components of color photographs. In the area of the zenith angle of $80^{\circ}$, near-horizontal maxima of sky brightness are observed, which, with increasing light wavelength, "shift" closer to the horizon. Fig. 4 shows that the zenith distance increases with the light wavelength increase. It is due to the decrease in the optical thickness of the atmosphere.

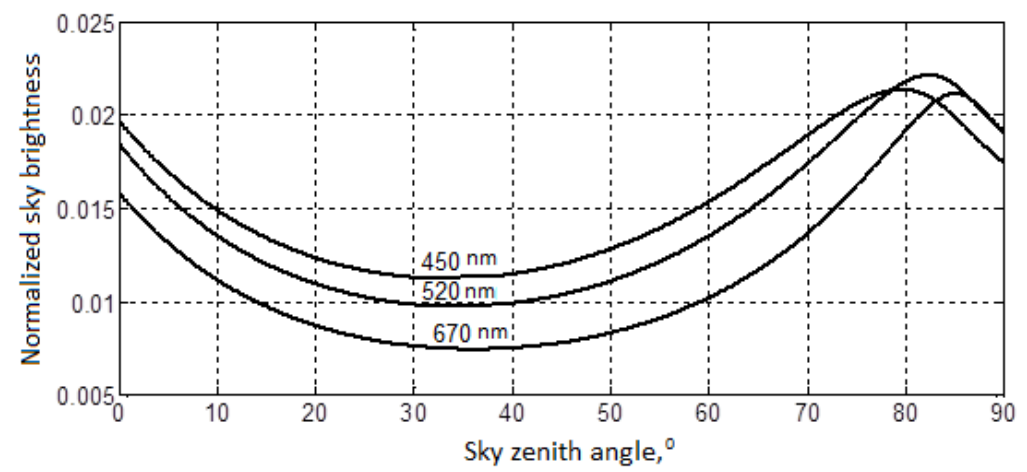

F i g. 3. The normalized sky brightness in the opposite to the sun direction as a function of the sky zenith angle $z$ for three light wavelengths 


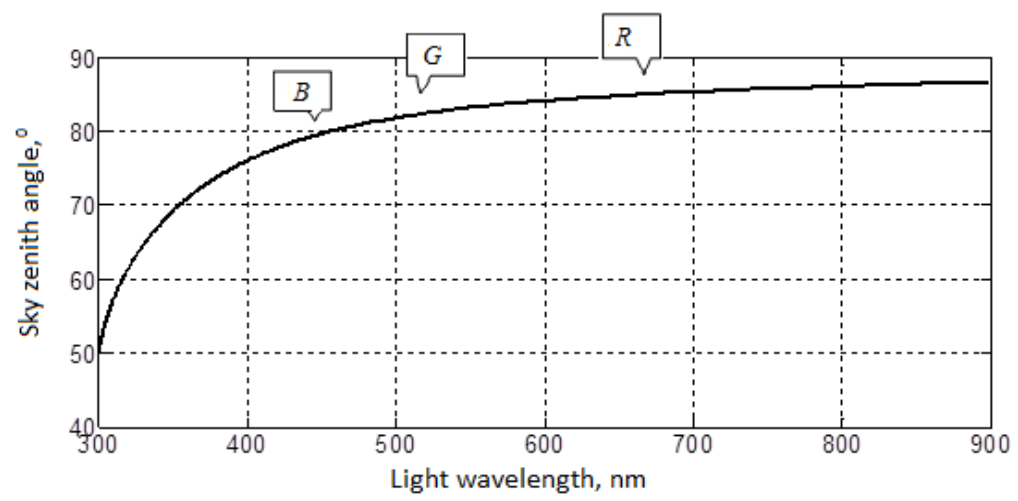

F i g. 4. Dependence of the zenith angle of the sky brightness near-horizon maximum upon the light wavelength

As calculations show, in the opposite to the sun part of the sky near the horizon, the angular dependence of the sky brightness is mainly determined by the factor $P\left(z, z_{s}\right)$ in the expression (1) for the sky brightness. Fig. 5 shows that a maximum value is observed near the horizon. Fig. 6 indicates that the dependencies shown there are almost the same. Thus, it can be concluded that approaching the horizon, the sky brightness first increases (Fig. 3) due to an increase in the length of the scattering path along the observation direction, but then decreases as a result of weakening the brightness of the sun rays on the way to the scattering path.

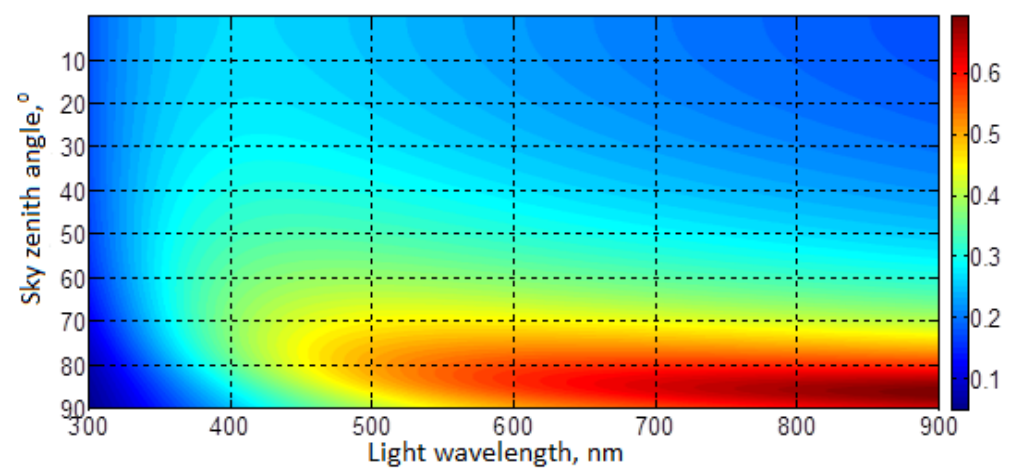

F i g. 5. Multiplier $P$ as a function of the zenith angle of the sky $z$ and the light wavelength (opposite to the sun direction of observation)

Digital cameras have good resolution, a large number of points in the images and allow determining the angular position of the near-horizontal maximum brightness for the $R, G$ and $B$-components of color images with good accuracy. Fig. 7 shows a near-horizontal maximum, which shifts towards the horizon as the wavelength of the light increases. The zenith distances at the horizontal maximum can be estimated as $81.4^{\circ}, 85.7^{\circ}$ and $87.8^{\circ}$ for the $B, G$ and $R$ components, respectively. By means of callouts, Fig. 4 shows the positions of these maxima. The points go higher, which may be due to different values of the atmospheric optical thickness in the experiment compared with those arbitrarily chosen for the model calculations. 


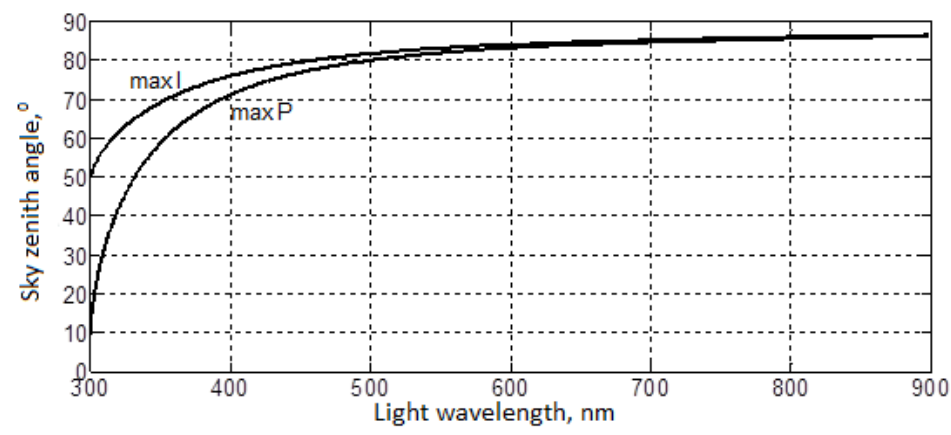

F i g. 6. Dependence of the zenith angle of the sky brightness near-horizon maximum upon the light wavelength for the sky brightness $I$ (upper curve) and the factor $P$ (lower curve)
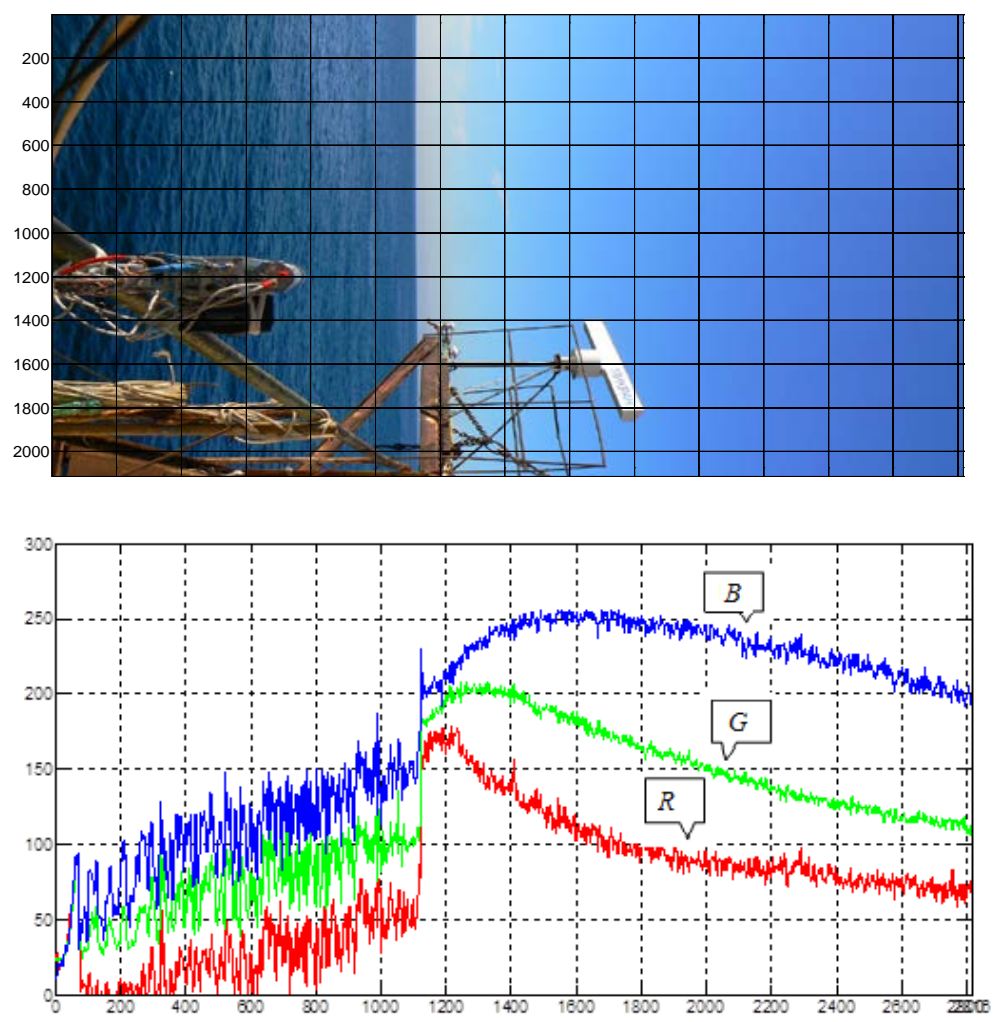

F i g. 7. Photo of the sky and the sea surface (above) made from the oceanographic platform in Katsiveli on 17.10.2014 at 10:17 (non-polarized light, photo dimensions: 2816 pixels (long side) $\times$ $\times 2112$ pixels), the $R, G, B$ horizontal sections (below) made in the middle of the photo in the point with the pixel number 1000 along the vertical axis. On the lower figure, the photo section value is shown on the vertical in the 8-bit scale. The upper and lower figures are superposed along the horizontal axis

The set forth approach to the analysis of the position of the near-horizontal maximum of the sky brightness further permits to find a solution to the inverse problem of estimating the optical thickness of the atmosphere from the position of the sky brightness maxima. 


\section{Algorithm for Estimating the Optical Thickness of the Atmosphere}

The following algorithm for estimating the optical thickness of the atmosphere for a certain value of the light wavelength $\lambda$ can be suggested. The Rayleigh thickness of the atmosphere $\tau_{r}$ is determined by the well-known formula (3), and the aerosol thickness of the atmosphere $\tau_{a}$ will be "searched" in a certain range of values in order to obtain the value of the calculated near-horizontal maximum, which coincides with the value obtained in the experiment. In this case, the zenith distance $z_{s}$ and the azimuthal distance of the sun relative to the observer $\psi$ should be taken into account in model calculations.

Fig. 8, where the normalized sky brightness in the opposite to the sun direction ( $\psi=180^{\circ}$ ) is shown as a function of the zenith angle of the sky point $z$ and the optical thickness of the atmosphere, the dependence of the zenith angle of the horizontal maximum brightness on the optical thickness is also shown. Fig. 9 on an enlarged scale presents a part of the dependence of the zenith angle of the nearhorizontal brightness maximum on the optical thickness at its small values. Here the arrow shows the value of the zenith angle at the horizontal maximum of brightness for the $G$-component of the image. This zenith angle corresponds to the optical thickness $\tau(520)=0.32$.

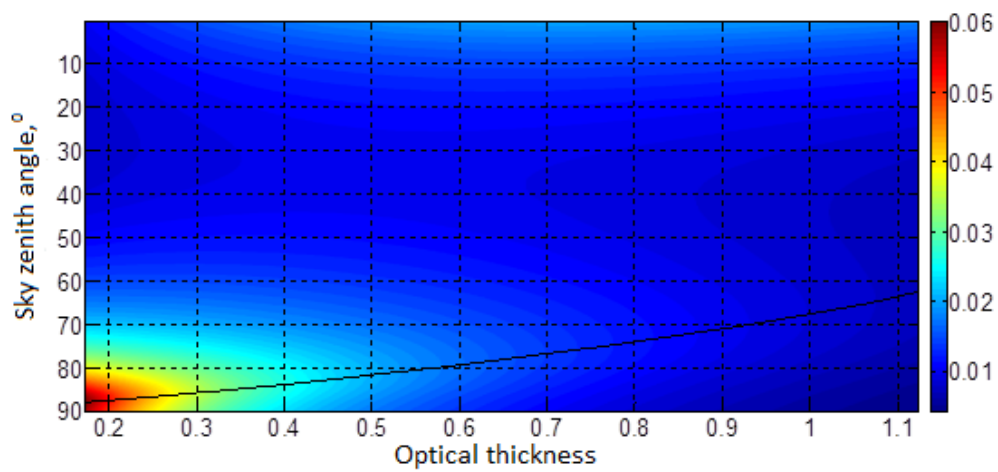

F i g. 8. The normalized sky brightness in the opposite to the sun direction as a function of the sky zenith angle $z$ and the optical thickness

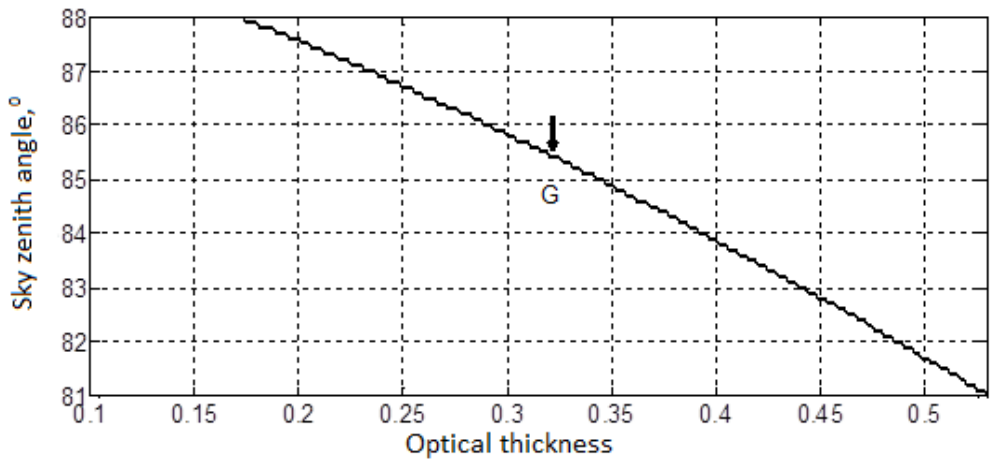

F i g. 9. Dependence of the zenith angle of the near-horizon brightness maximum upon the optical thickness, $\psi=180^{\circ}$ 
A refined analysis of the observation conditions showed that the image (Fig. 7) was taken when the azimuth angle of the sun relative to the direction of observation $\psi$ was close to $90^{\circ}$. Fig. 10, 11 shows the sky brightness distribution and the dependence of the zenith angle of the near-horizontal brightness maximum on the optical thickness at $\psi=90^{\circ}$. The following values of optical thickness $\left(z_{s}=60^{\circ}\right.$, $\psi=90^{\circ}$ ) were obtained:

$$
\tau_{r}(520)=0.123, \tau_{a}(520)=0.152, \tau(520)=0.275 .
$$

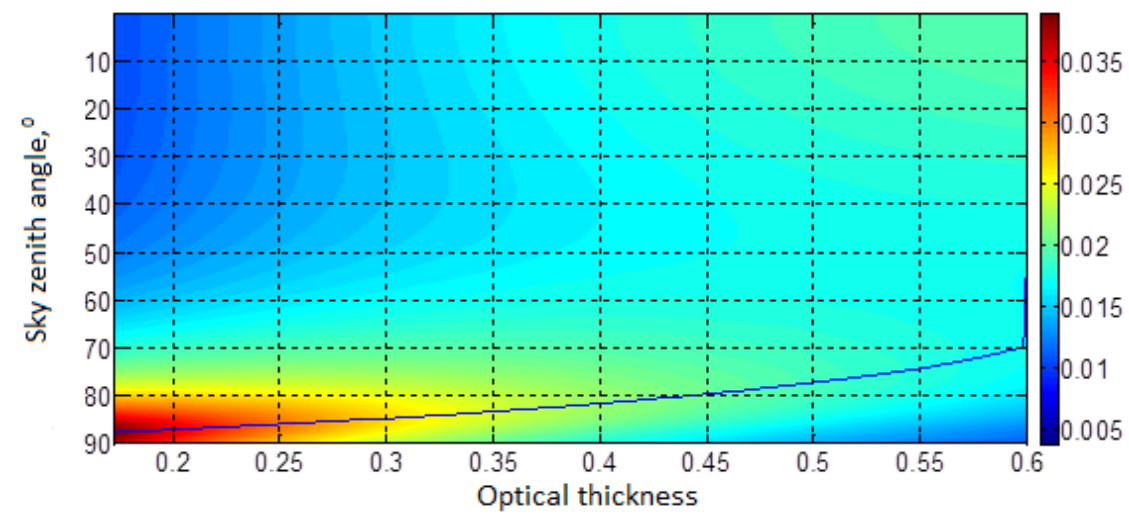

F i g. 10. The normalized sky brightness as a function of the sky zenith angle $z$ and the optical thickness. The curve showing dependence of the zenith angle of the near-horizon brightness maximum upon the optical thickness, $\psi=90^{\circ}$ is below

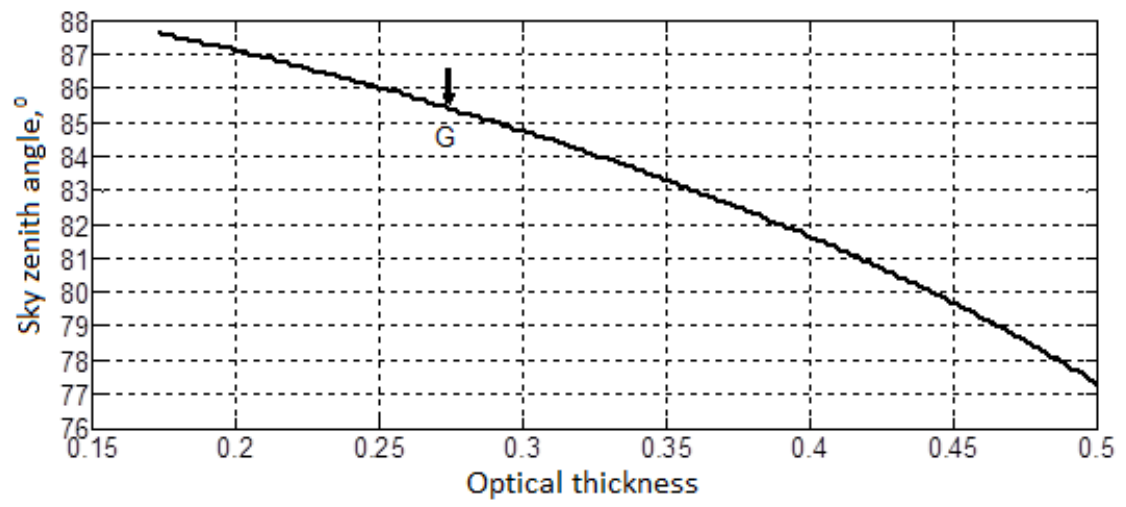

F i g. 11. Dependence of the zenith angle of the near-horizon brightness maximum upon the optical thickness, $\psi=90^{\circ}$

Note that with the sun azimuth variation, the estimate of the optical thickness does not change significantly: the difference in the estimate of the optical thickness for the sun azimuth of $180^{\circ}$ and $90^{\circ}$ is about $15-20 \%$. The proposed algorithm for recovering the atmospheric optical thickness values is "stable" to possible variations (errors) in determining the azimuthal position of the sun. 
Similar calculations were made for the $B$ and $R$ components of the image (Fig. 7); the following estimates of the optical thickness were obtained with their help $\left(z_{s}=60^{\circ}, \psi=90^{\circ}\right)$ :

$$
\begin{aligned}
& \tau_{r}(450)=0.2196, \tau_{a}(450)=0.2004, \tau(450)=0.42, \\
& \tau_{r}(670)=0.0447, \tau_{a}(670)=0.1153, \tau(670)=0.16 .
\end{aligned}
$$

Fig. 12 shows the optical thickness of the atmosphere for three light wavelengths. The obtained estimates of optical thicknesses coincide with the known results of field measurements of similar characteristics $[4,16]$.

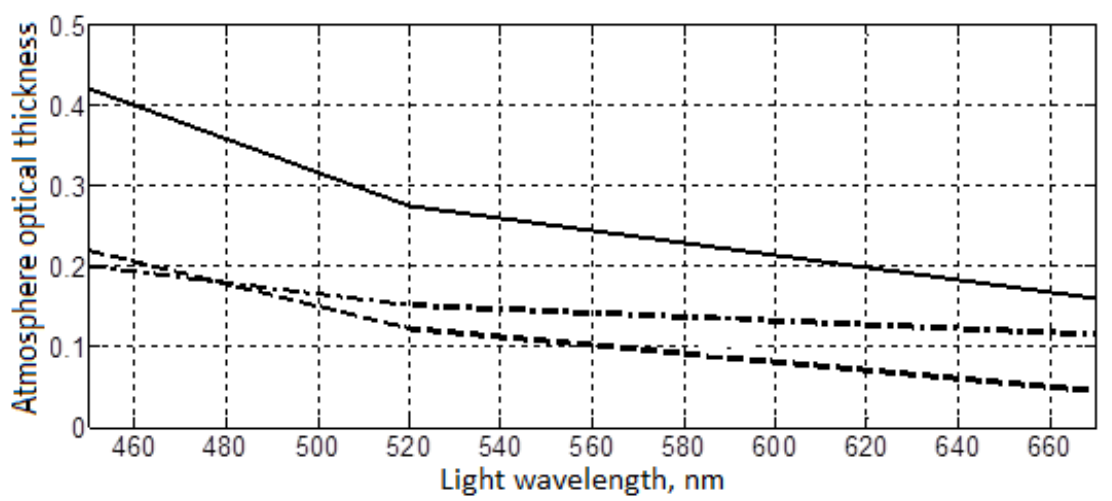

F i g. 12. The atmosphere optical thickness retrieved from the photo in Fig. 7 for three light wavelengths: 450, 520 and $670 \mathrm{~nm}$. The dash line denotes the Rayleigh optical thickness, the dash-dotted line - the aerosol optical thickness, the solid one is the sum of the aerosol and the Rayleigh optical thicknesses

It should be noted that a single approximation to describe the light scattering in the atmosphere is valid for small values of the optical thickness of the atmosphere. For large optical thicknesses in the ultraviolet range, light scattering of higher multiplicities should be taken into account. The set forth approach to the restoration of the optical thickness of the atmosphere permits to develop the used model of sky brightness taking into account the scattering of higher multiplicities, the albedo of the underlying surface, the "exponential" model of the atmosphere and the light polarization. This method of estimating the optical thickness of the atmosphere can be applied to restore the wave characteristics from space-time images of the sea surface in different spectral ranges of light [1]. Space-time images are formed over one-dimensional optical "sections" of the sea surface with a "capture" of the horizon and the near-surface portion of the sky, when the near-horizontal maximum of sky brightness is also recorded.

The method developed in the present paper makes it possible to obtain realtime estimates of the optical thicknesses of the atmosphere for three spectral ranges of light $R, G$ and $B$ from images of the near-horizontal section of the sky. The obtained values of the optical thickness can be applied in models of the angular distribution of the brightness of the cloudless sky to estimate the statistical characteristics of waves by the optical method, as well as to study the optical properties of the atmosphere. 


\section{REFERENCES}

1. Titov, V.I., Artamonov, A.Yu., Bakhanov, V.V., Ermakov, S.A., Luchinin, A.G., Repina, I.A. and Sergievskaja, I.A., 2014. Monitoring Sostojanija Poverhnosty Morja po ProstranstvennoVremennim Opticheskim Izobrajenijam [Monitoring of Sea Surface with Optical Technique]. Issledovanie Zemli iz Kosmosa, (5), pp. 3-14. doi:10.7868/S0205961414050078 (in Russian).

2. Bakhanov, V.V., Demakova, A.A., Korinenko, A.E., Ryabkova, M.S. and Titov, V.I., 2018. Estimation of the Wind Wave Spectra with Centimeters-to-Meter Lengths by the Sea Surface Images. Physical Oceanography, [e-journal] 25(3), pp. 177-190. doi:10.22449/1573-160X2018-3-177-190

3. Titov, V.I., Bakhanov, V.V., Ermakov, S.A., Luchinin, A.G., Repina, I.A. and Sergievskaya, I.A., 2014. Remote Sensing Technique for Near-Surface Wind by Optical Images of Rough Water Surface. International Journal of Remote Sensing, [e-journal] 35(15), pp. 5946-5957. doi:10.1080/01431161.2014.948223

4. Kokhanovsky, A.A., 2008. Aerosol Optics. Berlin: Springer, 154 p. https://doi.org/10.1007/978-3540-49909-1

5. Chapman, R.D., 1981. Visibility of RMS Slope Variations on the Sea Surface. Applied Optics, [e-journal] 20(11), pp. 1959-1966. https://doi.org/10.1364/AO.20.001959

6. Livshitz, G.Sh., 1973. Rasseyanij Svet Dnevnogo Neba [The Scattered Light of a Day Sky]. Alma-Ata: Nauka, 148 p. (in Russian).

7. Chapman, R.D. and Irani, G.B., 1981. Errors in Estimating Slope Spectra from Wave Images. Applied Optics, [e-journal] 20(20), pp. 3645-3652. https://doi.org/10.1364/AO.20.003645

8. Lee, R.L., 1994. Horizon Brightness Revisited: Measurements and a Model of Clear-Sky Radiances. Applied Optics, [e-journal] 33(21), pp. 4620-4628. https://doi.org/10.1364/AO.33.004620

9. Kokhanovsky, A.A., Breon, F.-M., Cacciari, A., Carboni, E., Diner, D., Nicolantonio, W.Di, Grainger, R.G., Grey, W.M.F., Höller, R. and Lee, K.-H [et al.], 2007. Aerosol Remote Sensing over Land: A Comparison of Satellite Retrievals Using Different Algorithms and Instruments. Atmospheric Research, [e-journal] 85(3-4), pp. 372-394. https://doi.org/10.1016/j.atmosres.2007.02.008

10. Lee, R.L. and Samudio, O.R., 2012. Spectral Polarization of Clear and Hazy Coastal Skies. Applied Optics, [e-journal] 51(31), pp. 7499-7508. https://doi.org/10.1364/AO.51.007499

11. Dubovik, O. and King, M.D., 2000. A Flexible Inversion Algorithm for Retrieval of Aerosol Optical Properties from Sun and Sky Radiance Measurements. JGR: Atmospheres, [e-journal] 105(D16), pp. 20673-20696. https://doi.org/10.1029/2000JD900282

12. Masuda, K., Sasaki, M., Takashima, T. and Ishida, H., 1999. Use of Polarimetric Measurements of the Sky over the Ocean for Spectral Optical Thickness Retrievals. Journal of Atmospheric and Oceanic Technology, [e-journal] 16(7), pp. 846-859. https://doi.org/10.1175/15200426(1999)016<0846:UOPMOT>2.0.CO;2

13. Mishchenko, M.I. and Travis, L.D., 1997. Satellite Retrieval of Aerosol Properties over the Ocean Using Polarization as Well as Intensity of Reflected Sunlight. JGR: Atmospheres, [e-journal] 102(D14), pp. 16989-17013. https://doi.org/10.1029/96JD02425

14. Wang, M. and Gordon, H.R., 1994. Estimating Aerosol Optical Properties over the Oceans with the Multiangle Imaging Spectroradiometer: Some Preliminary Studies. Applied Optics, [e-journal] 33(18), pp. 4042-4057. https://doi.org/10.1364/AO.33.004042

15. Bazalitskaya, G.P. and Livshits, G.S., 1982. Funktsii Rasseyania Sveta v Bezoblachnoj Atmosfere [Light Scattering Functions in a Cloudless Atmosphere]. Izvestiya Akademii Nauk SSSR: Fizika Atmosfery i Okeana, 18(5), pp. 551-555 (in Russian).

16. Dolin, L.S. and Levin, I.M., 1991. Spravochnik po Teorii Podvodnogo Videnia [Handbook in the Theory of Underwater Vision]. Leningrad: Gidrometeoizdat, 229 p. (in Russian). 
17. Hasekamp, O.P. and Landgraf, J., 2007. Retrieval of Aerosol Properties over Land Surfaces: Capabilities of Multiple-Viewing-Angle Intensity and Polarization Measurements. Applied Optics, [e-journal] 46(16), pp. 3332-3344. https://doi.org/10.1364/AO.46.003332

18. Deuzé, J.L., Bréon, F.M., Devaux, C., Goloub, P., Herman, M., Lafrance, B., Maignan, F., Marchand, A., Nadal, F., Perry, G. and Tanré, D., 2001. Remote Sensing of Aerosols over Land Surfaces from POLDER-ADEOS-1 Polarized Measurements. JGR: Atmospheres, [e-journal] 106(D5), pp. 4913-4926. https://doi.org/10.1029/2000JD900364

About the authors:

Viktor V. Bakhanov - Head of the Laboratory of Hydrophysical and Acoustic Modeling, IAP RAS (46 Ul'yanov Street, Nizhny Novgorod, 603950, Russia), Ph.D. (Phys.-Math.), Scopus Author ID: 6603623205, bakh@hydro.appl.sci-nnov.ru

Anastasiya A. Demakova - Research Assistant, postgraduate, IAP RAS (46 Ul'yanov Street, Nizhny Novgorod, 603950, Russia), d6365@yandex.ru

Viktor I. Titov - Senior Research Associate, IAP RAS (46 Ul'yanov Street, Nizhny Novgorod, 603950, Russia), Ph.D. (Phys.-Math.), Scopus Author ID: 7201990965, titov@hydro.appl.sci-nnov.ru

Contribution of the co-authors:

Viktor V. Bakhanov - scientific leadership, discussion of article

Anastasiya A. Demakova - experimental research, processing of experimental data cle

Viktor I. Titov - scientific leadership, processing of experimental data, preparation of the arti-

All the authors have read and approved the final manuscript.

The authors declare that they have no conflict of interest. 